\title{
Comparison of Levosimendan Versus IABP in Patients with Poor Left Ventricular Function Undergoing Coronary Artery Bypass Graft Surgery
}

\author{
Alaa Omar, MD, Mahmoud Eldegwy, MD, Mohamed Allam, MD, Amr Rouchdy, MD, Soliman Abdel Hei \\ Soliman, MD, Omar Dawoud, MD, Ehab Elshihy, MD \\ Cardiothoracic Surgery Department, Faculty of Medicine, Cairo University, Cairo, Egypt
}

\section{ABSTRACT}

Objectives: The aim of this study was to compare the use of levosimendan versus intra-aortic balloon pump (IABP) in patients with poor left ventricular function undergoing coronary artery bypass grafting (CABG) with ejection fraction less than $35 \%$.

Methods: Between February 2016 and March 2019, a prospective randomized study was performed on a group of 279 consecutive patients with left ventricular ejection fraction $<35 \%$, who underwent elective CABG without concomitant procedures. These patients were divided into 2 groups, according to the treatment they received - either levosimendan (Group A) or intra-aortic balloon counterpulsation (Group B).

Results: There was no statistically significant difference between the 2 groups, regarding mortality and morbidity. In the IABP group, the mean arterial blood pressure (2 hours post cardiopulmonary bypass) significantly was higher, and the heart rate in postoperative Day 1 significantly was lower. The levosimendan group had a significantly lower ICU stay than the IABP group.

Conclusion: We found that starting levosimendan infusion after induction of anesthesia is an acceptable option in comparison to IABP. The use of levosimendan in high-risk cardiac patients is comparable to IABP in improving hemodynamics during and after conventional on-pump CABG and results in a shorter ICU stay.

\section{INTRODUCTION}

In recent years, cardiac surgery patients are characterized by older age, more preoperative co-morbidities, and jeopardized left ventricular function, the so-called "high risk" patients [Miceli 2009]. To decrease the risk, intra-aortic balloon pump (IABP) is a form of internal counter pulsation that has been used as an assisting circulatory support device. In diastole augmentation due to inflation of IAB potentially increases cerebral, coronary and systemic circulations

Received September 5, 2019; accepted fanuary 2, 2020.

Correspondence: Alaa Omar, MD, Assistant Professor of Cardiothoracic Surgery, Cardiothoracic Surgery Department, Faculty of Medicine, Cairo University, 14 Mossaddak St.Dokki, 6th floor, Cairo, Egypt; (e-mail: alaaomarcts1@yahoo.com).
[Parissis 2016]. Indications of perioperative insertion of intra-aortic balloon pump in cardiac surgery is well known and supported in the literature [Myat 2012; Toller 2013]. Traditionally dopamine, dobutamine and epinephrine have been used as inotropic support in cardiac surgery [Hardy 1993]. The mechanism of action of most currently available inotropic drugs is by augmenting myocardial contractility through enhancing concentrations of intracellular calcium leading to an increase in myocardial oxygen consumption [Silva-Cardoso 2009]. With the use of levosimendan, occurrence of arrhythmias is reduced. This can be attributed to the fact that total intracellular calcium levels are not raised. Levosimendan does not affect the duration of diastole and so ventricular relaxation is not jeopardized, therefore maintaining adequate ventricular filling and optimal coronary perfusion. Vasodilatation occurring through the opening of potassium channels may predispose hypotension [Packer 2013]. Long-term benefits are noted with levosimendan use, as the presence of a pharmacologically active metabolite with a long elimination half-life (75-80 h) leads to long standing hemodynamic effects lasting up to 7 to 9 days [Altenberger 2010].

\section{MATERIALS AND METHODS}

This research was performed after informed consent was obtained from each patient as well as approval from our department council and ethical committees. Between February 2016 and March 2019, a prospective randomized study was performed on a group of 279 consecutive patients with left ventricular ejection fraction $<35 \%$, who underwent elective CABG without concomitant procedures. Excluded were unstable cases undergoing urgent CABG, recent acute myocardial infarction (within 2 weeks before surgery). Also excluded from our study were patients with severe chronic obstructive pulmonary disease (COPD), renal failure requiring hemodialysis, and history of cerebrovascular stroke.

Patients included in the study presented with multiple coronary vessels disease indicated for CABG confirmed by coronary angiographic studies, and those with preoperative echocardiography that revealed poor left ventricular function with ejection fraction less than $35 \%$. Exclusion criteria were those who had levosimendan administered within the preceding 30 days; patients with contraindications to IABP (ie, severe peripheral vascular disease, aortic regurgitation, dissection, or aneurysm); patients with presence of significant ischemic 
Table 1. Demographic data and clinical characteristics of the patients

\begin{tabular}{lccc}
\hline Variable & Levosimendan (Group A) & IABP (Group B) & $P$ \\
\hline Male/Female & $98 / 37(73 \% / 27 \%)$ & $120 / 24(83 \% / 17 \%)$ & .54 \\
Age & $57.7 \pm 4.8$ & $58.8 \pm 4.2$ & .34 \\
BMI & $30 \pm 3.4$ & $29 \pm 3.5$ & .32 \\
DM & $76(56 \%)$ & $91(63 \%)$ & .65 \\
Hypertension & $81(60 \%)$ & $101(70 \%)$ & .52 \\
EF & $32.2 \pm 2.1 \%$ & $33 \pm 1.7$ & .07 \\
NYHA class & $11(8 \%)$ & $7(5 \%)$ & \\
Class II & $108(80 \%)$ & $125(87 \%)$ & .35 \\
Class III & $16(12 \%)$ & $12(8 \%)$ & \\
Class IV & $5.8 \pm 1.1$ & $5.2 \pm 2$ & .10 \\
Euroscore & & & \\
\hline
\end{tabular}

Continuous variables are expressed as mean \pm SD and categorical variables as number (\%)

$P<.05=$ significant.

mitral regurge $>+1$ grade; and those who were CABG reoperations, had chronic obstructive pulmonary disease, or liver and kidney diseases.

The primary end point was mortality. The secondary end points were ICU and hospital stays, mechanical ventilation time, morbidities (arrhythmias, dialysis, reopening, and mediastinitis), and postoperative ejection fraction.

There were no significant differences in demographic data or baseline clinical characteristics between the groups, as shown in the first table (Table 1).

In all cases, history taking and physical examination as well as complete laboratory investigation, chest $\mathrm{x}$-ray, 2D and $M$ mode echocardiography, and myocardial viability study (if available) were reviewed. Ejection fraction was estimated using Simpson's method by 2D transthoracic echocardiography. All patients had a Swan-Ganz catheter inserted through the right internal jugular vein and hemodynamics of patients meticulously were revised. Cardiac index was estimated using Swan-Ganz catheter.

In Group A, levosimendan was administered intravenously through a central venous line after induction of anesthesia. Hemodynamics closely were monitored with a continuous infusion of $0.1 \mu \mathrm{g} / \mathrm{kg} / \mathrm{min}$ diluted in $5 \%$ glucose with a $0.25 \mathrm{mg} / \mathrm{mL}$ concentration over 24 hours (without boluses).

In the IABP group (Group B), insertion of the device was done with induction of general anesthesia. The IABP was inserted through the femoral artery using Seldinger technique under local anesthesia and sedation (Datascope Sensation, linear 7.5 F, 40 mL; Datascope Corp, Fairfield, NJ). Sheathless insertion was the preferred method. Heparin infusion was initiated postoperatively in the ICU after confirming the absence of significant mediastinal bleeding at a rate of $5 \mathrm{U} / \mathrm{kg} / \mathrm{h}$ to maintain ACT within $150 \mathrm{sec}$. The IABP was kept for at least 36 hours postoperatively or until the patient's hemodynamics and parameters showed no finding of low cardiac output. Routine median sternotomy incision was performed in all cases. Standard cardiopulmonary bypass (CPB) was initiated through aorto-atrial cannulation in all patients after assuring that the ACT was above $480 \mathrm{sec}$. Left internal mammary artery almost always was grafted to the left anterior descending artery, while the great saphenous vein was used to graft other targets. Warm intermittent antegrade blood potassium cardioplegia was used for myocardial protection. Heparin was reversed with protamine sulfate after weaning from CPB. In the IABP group, epinephrine and/or norepinephrine infusion were started if needed to keep the cardiac index above $2.0 \mathrm{~L} / \mathrm{min} / \mathrm{m}^{2}$. In the levosimendan group, norepinephrine almost always was used to avoid severe peripheral vasodilatation. With nearly all patients in both groups, we used norepinephrine to avoid the peripheral vasodilatory effect of both levosimendan and IAB to maintain sufficient hemodynamics. When necessary, epinephrine was added to maintain cardiac index above $2.0 \mathrm{~L} / \mathrm{min} / \mathrm{m}^{2}$.

Statistical analysis was performed with SPSS software v. 24. All data are expressed as mean and standard deviation. Categorical variables are expressed as number and percentage. $P$ value $<.05$ was considered statistically significant.

\section{RESULTS}

Regarding intraoperative data, the total bypass time was calculated for all patients. The mean bypass time in Group A ranged between $60-135$ minutes with a mean $85.4 \pm 20.99$ minutes. In Group B, it ranged between 67-133 minutes with a mean $90.7 \pm 20.4$ minutes. The number of grafts ranged from 2 to 4 with a mean of $3.2 \pm 0.48$ in Group A, while Group $B$ had a number of grafts ranging from 3 to 4 with a mean of $3.3 \pm 0.47$. There was no statistical significance between the 2 groups in both bypass time and number of grafts.

In our study, we recorded the mean arterial pressure, central venous pressure, heart rate, and cardiac index of patients in the 2 groups as shown in the second table (Table 2). The most important finding after analysis of the hemodynamic parameters of the patients in the 2 groups was the statistically significant difference in the mean arterial pressure at 2 hours after CPB. It was $79.8 \pm 7.9$ in Group A compared with 84.8 \pm 8.7 in Group B $(P=.02)$. Also, the heart rate showed statistically significant difference in postoperative day 1 (POD 1) being $90.6 \pm 9.3$ in Group A compared with $83.3 \pm 6.8$ in Group B $(P=.05)$.

The postoperative data shown in Table 3 , revealed no statistically significant difference between the 2 groups, regarding postoperative mechanical ventilation time, arrhythmias, reopening, need for hemodialysis, mediastinitis, or hospital stay (Table 3).

We used norepinephrine with nearly all patients to avoid peripheral vasodilatatory effects in both groups to maintain cardiac index above $2 \mathrm{~L} / \mathrm{min} / \mathrm{m}^{2}$, and we added adrenaline when needed to maintain sufficient hemodynamics. There was no statistically significant difference in both groups, 
Table 2. Perioperative hemodynamic parameters

\begin{tabular}{|c|c|c|c|c|c|c|c|c|}
\hline Variable & Levo & IABP & Levo & IABP & Levo & IABP & Levo & $\mathrm{IABP}$ \\
\hline Mean arterial pressure $(\mathrm{mmHg})$ & \multicolumn{2}{|c|}{$P=.08(\mathrm{NS})$} & \multicolumn{2}{|c|}{$P=.02($ Significant $)$} & \multicolumn{2}{|c|}{$P=.74(\mathrm{NS})$} & \multicolumn{2}{|c|}{$P=.76(\mathrm{NS})$} \\
\hline Central venous pressure $(\mathrm{mmHg})$ & $10.1 \pm 1.9$ & $9.7 \pm 1.9$ & $10.4 \pm 1.9$ & $9.8 \pm 1.8$ & $9.3 \pm 2$ & $8.9 \pm 1.8$ & $9 \pm 1$ & $8.6 \pm 1.1$ \\
\hline \multirow[t]{2}{*}{ Heart rate (beat/min) } & $67.5 \pm 6.6$ & $68.4 \pm 10.5$ & $80.2 \pm 8.5$ & $82.4 \pm 10.5$ & $84.6 \pm 9.1$ & $78.3 \pm 11.5$ & $90.6 \pm 9.3$ & $83.3 \pm 6.8$ \\
\hline & \multicolumn{2}{|c|}{$P=.67(\mathrm{NS})$} & \multicolumn{2}{|c|}{$P=.42(N S)$} & \multicolumn{2}{|c|}{$P=.34(\mathrm{NS})$} & \multicolumn{2}{|c|}{$P=.008$ (Significant) } \\
\hline \multirow[t]{2}{*}{ Cardiac index $\left(\mathrm{l} / \mathrm{min} / \mathrm{m}^{2}\right)$} & $1.4 \pm 0.2$ & $1.5 \pm 0.38$ & $1.6 \pm 0.2$ & $1.6 \pm 0.2$ & $2 \pm 0.8$ & $2.2 \pm 0.9$ & $2.5 \pm 0.9$ & $2.6 \pm 0.92$ \\
\hline & \multicolumn{2}{|c|}{$P=.13(\mathrm{NS})$} & \multicolumn{2}{|c|}{$P=.09(\mathrm{NS})$} & \multicolumn{2}{|c|}{$P=.18(N S)$} & \multicolumn{2}{|c|}{$P=.08(N S)$} \\
\hline
\end{tabular}

regarding use of inotropes; with both, we almost always used norepinephrine and there was no difference in use of epinephrine (0.68). We found no significant difference between the 2 groups in mean time of inotropic support with 2.3 days in Group A and 2.6 days in Group B (0.65). Regarding mortality, 9 patients died in Group A - one from acute renal failure and 8 from multi-organ failure, due to low cardiac output syndrome. In Group B, 12 patients died - 2 patients developed acute renal failure and the remaining 10 patients died from multi-organ failure, due to low cardiac output syndrome. There also was no statistically significant difference between the 2 groups regarding mortality. The postoperative cardiac function was measured by estimating the ejection fraction (EF $\%)$. In Group A, the patients had an ejection fraction, ranging from $30-49 \%$ with a mean of $34.2 \pm 2.1$, while in Group B, it ranged from $31-50 \%$ with a mean of $38.7 \pm 4.6 \%$. There was no statistical significance between the 2 groups.

The only postoperative parameter showing statistical significance between the 2 groups was intensive care unit (ICU) stay. In Group A, it ranged between 3 to 6 days with a mean of $4.4 \pm 0.8$ days, while Group B ranged from 4 to 7 days with a mean of $5.2 \pm 0.9$ days $(P=.05)$.

In Group B (IABP), 6 patients developed limb ischemia $(4.1 \%)$. Five of those patients had transient ischemia that resolved after removal of IABP, while the remaining patient required a vascular surgical intervention in the form of fasciotomy. Bleeding with hematoma occurred in 4 patients $(2.8 \%)$ without need for surgical intervention.

\section{DISCUSSION}

In the last decade, the great innovation in the treatment modalities of coronary artery disease with interventional cardiology provided a less invasive option than surgery in treatment of cardiac patients, changing the cohort of patients referred to cardiac surgery into high-risk cases [Miceli 2009]. The available surgical patients categorized as high risk are characterized by multiple co-morbidities, older age, lower cardiac function, and worse clinical condition compared to before. Ischemic dilated cardiomyopathy is considered a big problem that surgeons today. This explains the increasing numbers of patients with (low cardiac output syndrome) LCOS both pre- and postoperatively with a very high mortality rate [Elahi 2011]. IABP is a frequently used method in cardiac surgery that provides a mechanical circulatory support by reducing myocardial oxygen consumption of the heart, decreasing after load and enhancing hemodynamic stability improving the results of high-risk patients with coronary artery disease undergoing surgery. Vascular injury, bleeding and limb ischemia are considered troublesome complications of IABP insertion [Lomivorotov 2011]. Preoperative prophylactic IABP improves outcome and reduced hospital mortality. A retrospective analysis done by Lavana and colleagues showed a reduction in hospital mortality in high-risk CABG patients [Lavana 2010].

Wang and colleagues [Wang 2007] demonstrated that preoperative prophylactic IABP provides a decrease in shortterm mortality in high-risk CABG patients with the same conclusion being reached by Suzuki and colleagues [Suzuki 2004]. Levosimendan is a calcium sensitizer drug that's effective in treatment of heart failure and also has protective properties on the heart as it facilitates opening of the adenosine triphosphate dependent potassium channel. Also, it decreases afterload, leading to increased cardiac index [Elahi 2011].

In Group A, levosimendan was administered through a central venous catheter for approximately 24 hours as a continuous infusion of $0.1 \mathrm{micrograms} / \mathrm{kg} / \mathrm{min}$ diluted in $5 \%$ glucose with a $0.25-\mathrm{mg} / \mathrm{mL}$ concentration, without boluses. Levosimendan considerably improved hemodynamics without increase in myocardial oxygen demand. Di Molfetta and colleagues showed superiority of levosimendan in comparison with other conventional inotropes. The timing of its administration was important. Preoperative administration to patients with severely reduced ventricular contractility is recommended [Di Molfetta 2018].

It was found that patients with a preoperative LVEF $\leq 30 \%$ who received levosimendan were treated with a smaller amount of dobutamine in comparison with those treated with milrinone and show a lower mortality rate after surgery [De Hert 2007].

Impaired LV function was a constant characteristic of all our patients who received levosimendan after induction of 
Table 3. Postoperative data in the study groups

\begin{tabular}{lccc}
\hline & $\begin{array}{c}\text { Levosimendan } \\
(\text { Group A) }\end{array}$ & $\begin{array}{c}\text { IABP } \\
\text { (Group B) }\end{array}$ & $P$ \\
\hline ICU stay (days) & $4.4 \pm 0.8$ & $5.2 \pm 0.9$ & .005 \\
MV time (hours) & $11.1 \pm 2.4$ & $11.9 \pm 3.3$ & .65 \\
Arrhythmia & $18(13 \%)$ & $14(10 \%)$ & .53 \\
Dialysis & $2(1 \%)$ & $4(3 \%)$ & .32 \\
Reopening & $6(4 \%)$ & $8(6 \%)$ & .57 \\
Mortality & $9(7 \%)$ & $12(8 \%)$ & .66 \\
Hospital stay (days) & $10.6 \pm 1.2$ & $11 \pm 1.1$ & .13 \\
Ejection fraction (\%) & $34.2 \pm 2.1$ & $38.7 \pm 4.6$ & .54 \\
Inotropes (adrenaline) & $44(33 \%)$ & $50(35 \%)$ & .68 \\
Mediastinitis & $20(15 \%)$ & $13(9 \%)$ & .07 \\
\hline
\end{tabular}

Continuous variables are expressed as mean \pm SD and categorical variables as number $(\%) P<.05=$ significant.

IABP: Intra-aortic balloon pump

ICU: Intensive care unit

MV: Mechanical ventilation

anesthesia as a prophylaxis against low cardiac output syndrome (LOS). Timing of levosimendan infusion could be of critical importance, when analyzing outcome for many authors. In a study where 45 patients with LOS received levosimendan either after induction of anesthesia or in the ICU, hemodynamic responses were similar in the 2 groups, but the anesthesia group had significantly shorter stay both in ICU and at the hospital [Tasouli 2007]. In a retrospective study by Lavana and colleagues, starting IABP before surgery may cause a reduction in hospital mortality [Lavana 2010].

The perioperative use of levosimendan in high-risk patients can decrease the ICU stay, when compared with the IABP. Patients with absolute contraindications to the IABP, such as severe peripheral arterial disease or aortic regurgitation also can benefit from this strategy. When compared with IAB, use of levosimendan infusion in this cohort of high surgical risk patients can lead to shorter ICU stay. Also, when there is an absolute contraindication for use of IAB as in patients with extensive peripheral vascular disease or those who had significant aortic regurgitation, they can benefit from levosimendan infusion.

It was found that the use of levosimendan in patients with massively jeopardized systolic function and symptoms of congestive heart failure undergoing heart surgery showed a dramatic augmentation in $\mathrm{CO}$ and improvement in various hemodynamic parameters [Rajek 2003].

Levosimendan facilitates weaning from CPB in high perioperative risk patients, decreased catecholamine needs, mechanical circulatory support, and ICU stay. But no data supports optimum dosage and timing for levosimendan administration [Shahzad 2006].

In our study, we used norepinephrine in almost always all patients to avoid peripheral vasodilatatory effects in both groups to maintain cardiac index above $2 \mathrm{~L} / \mathrm{min} / \mathrm{m}^{2}$. We added adrenaline, when needed to maintain sufficient hemodynamics. There was no statistically significant difference in the groups, regarding use of inotropes as we nearly always used norepinephrine, and there was no difference in use of epinephrine (0.68). There was no significant difference between the 2 groups in mean time of inotropic support of 2.3 days in Group A and 2.6 days in Group B (0.65). We did not use neosyephrein as it is not available, and our center's protocol is to avoid vasopressin use in CABG patients. This is because of possible coronary vasospasm, which we encountered with some previous patients who underwent CABG; after neosyephrein use, it led to significant arrhythmias that sometimes were life threatening and unpredictable. We only use neosyephrein in CABG patients, if there is severe vasoplegia not controlled with both epinephrine and norepinephrine and only with great caution. We frequently use it if vasoplegia has occurred in non-CABG patients with satisfactory outcome. Vasopressin can induce both systemic and coronary vasoconstriction basically through the vasopressin $\mathrm{V} 1$ receptor and can decrease coronary blood flow via the constriction of small coronary vessels. Studies have shown that ST-segment elevation ECG changes can occur with use of vasopressin, due to its induced coronary vasospasm without underlying significant coronary artery ischaemic lesions [Peberdy 2010].

In our study, we noticed significantly higher heart rate at postoperative Day 1 in the levosimendan group. This comes in favor with the previous studies concluding the potent inotropic and vasodilator effect of levosimendan. In the levosimendan group compared with the IABP group, mean arterial blood pressure significantly was lower at 2 hours post CPB. However, mortality and the rate of other major complications showed no statistically significant difference between the 2 groups. Some investigators found that levosimendan was associated with an increased risk of postoperative atrial fibrillation [Ayman 2018], but in our study no statistically significant difference regarding arrhythmias was found.

\section{CONCLUSION}

We found that starting levosimendan infusion after induction of anesthesia is an acceptable option in comparison to IABP. The use levosimendan in high-risk cardiac patients is comparable to IABP, in terms of improving hemodynamics during and after conventional on-pump CABG and it resulted in shorter ICU stay.

\section{REFERENCES}

Altenberger J, Parissis JT, Ulmer H, Poelzl G. 2010. Rationale and design of the multicentre randomized trial investigating the efficacy and safety of pulsed infusions of levosimendan in outpatients with advanced heart failure (LevoRep study). Eur J Heart Fail. 12:186-192.

De Hert SG, Lorsomradee S, Cromheecke S, Van der Linden PJ. 2007. The effects of levosimendan in cardiac surgery patients with poor left ventricular function. Anesth Analg. 104:766-73. 
Di Molfetta P, Gregorini R, Fiore C, Santarpino G. 2018. Is There Still Room for the Prophylactic Use of Levosimendan in Cardiac Surgery? Ann Thorac Surg 106:1590-5.

Elahi M, FRCS CTh, PhD, Lam J, MBBS, Asopa S, MRCS, PhD, Matata BM, FRSMed, PhD. 2011. Levosimendan Versus an Intra-aortic Balloon Pump in Adult Cardiac Surgery Patients With Low Cardiac Output Journal of Cardiothoracic and Vascular Anesthesia, Vol 25, No 6 (December) pp 1154-1162.

Elbadawi A, MD, Elgendy IY, MD, Saad M, MD, PhD, Megaly M, MD, MS, Mentias A, MD, Abuzaid AS, MD, Shahin HI, MS, Goswamy V, MD, Abowali H, MD, London B, MD. 2018. Meta-Analysis of Trials on Prophylactic Use of Levosimendan in Patients Undergoing Cardiac SurgeryAnn Thorac Surg 105:1403-10.

Hardy JF, Belisle S. 1993. Inotropic support of the heart that fails to successfully wean from cardiopulmonary bypass. The Montreal Heart Institute experience. J Cardiothorac Vasc Anesth 7(suppl 2):33-39.

Lavana JD, Fraser JF, Smith SE, Drake L, Tesar P and Mullany DV. 2010. Influence of timing of intra-aortic balloon placement in cardiac surgical patients. J Thorac Cardiovasc Surg 140: 80-85.

Lomivorotov VV, Cherniavskiy A, Boboshko V, Kornilov I, Lomivorotov VN, Karaskov A. 2011. Levosimendan vs. intra-aortic balloon pump in high-risk cardiac surgery. Asian Cardiovascular \& Thoracic 19(2) 154-159.

Miceli A, Fiorani B, Danesi TH, Melina G, Sinatra R. 2009. Prophylactic intra-aortic balloon pump in high-risk patients undergoing coronary artery bypass grafting: a propensity score analysis. Interact CardioVasc Thorac Surg 9(2):291-294.

Myat A, McConkey H, Chick L, Baker J, Redwood S. 2012. The Intraaortic Balloon Pump in High-risk Percutaneous Coronary Intervention, Cardiol 4(2):211-234.

Packer M, Wilson C, Fisher L, Massie BM, Teerlink JR, Young J, Robert JP, Thakkar R, Delgado-Herrera L, Salon J, Garratt C, Huang B, Sarapohja T. 2013. Effect of Levosimendan on the Short-Term Clinical Course of Patients With Acutely Decompensated Heart Failure. JCHF. $1: 103-111$.
Parissis H, Graham V, Lampridis S, Lau M, Hooks G, Mhandu PC. 2016. IABP: history-evolution-pathophysiology indications: what we need to know , Journal of Cardiothoracic Surgery 11:122.

Peberdy MA, Callaway CW, Neumar RW, et al. 2010. Part 9: post-cardiac arrest care: 2010 American Heart Association guidelines for cardiopulmonary resuscitation and emergency cardiovascular care. Circulation 122 Suppl 3:S768-S786.

Raja SG, MRCS, and Rayen BS, DCH. 2006. Levosimendan in Cardiac Surgery: Current Best Available Evidence Ann Thorac Surg 81:1536-46.

Rajek AM, Koinig H, Jelen M, Schiferer, Hutschala D. 2003. Levosimendan, a new Ca-sensitizer, in patients with poor left ventricular function undergoing cardiac surgery Anesthesiology 99:A133.

Silva-Cardoso J, Ferreira J, Oliveira-Soares A, Martins-de-Campos J, Fonseca C, Lousada N, Ilídio-Moreira J, Rabaçal C, Damasceno A, Amorim S, Seabra-Gomes R, Ferreira R, Abreu-Lima C. 2009. Effectiveness and safety of levosimendan in clinical practice. Rev Port Cardiol 28: $143-54$.

Suzuki T, Okabe M, Handa M, et al. 2004. Usefulness of preoperative intraaortic balloon pump therapy during off-pump coronary artery bypass grafting in high-risk patients. Ann Thorac Surg 77:2056-9.

Tasouli A, Papadopoulos K, Antoniou T, Kriaras I, Stavridis G, Degiannis D, Geroulanos S. 2007. Efficacy and safety of perioperative infusion of levosimendan in patients with compromised cardiac function undergoing open-heart surgery: importance of early use. Eur J Cardiothorac Surg 32: 629-633.

Toller W, Algotsson L, Guarracino F, Hörmann C, Knotzer J, Lehmann A, Rajek A, Salmenperä M, Schirmer U, Tritapepe L, Weis F, Landoni G. 2013. Perioperative use of levosimendan: best practice in operative settings. J Cardiothorac Vasc Anesth 27:361-366.

Wang J, MD, Yu W, MD, Gao M, MD, Gu C, MD, Yu Y, MD, PhD. 2016. Preoperative Prophylactic Intraaortic Balloon Pump Reduces the Incidence of Postoperative Acute Kidney Injury and Short-Term Death of High-Risk Patients Undergoing Coronary Artery Bypass Grafting: A Meta-Analysis of 17 Studies Ann Thorac Surg 101:2007-19. 